Pierre-Alain Duc, Jonathan Braine and Elias Brinks, eds.

\title{
Intracluster Planetary Nebulae in Clusters and Groups
}

\author{
John J. Feldmeier \\ Department of Astronomy, Case Western Reserve University, \\ Cleveland, $\mathrm{OH}, \mathrm{USA}$ \\ Robin B. Ciardullo \\ Department of Astronomy and Astrophysics, Pennsylvania State \\ University, University Park, PA, USA
}

George H. Jacoby

WIYN Observatory, Tucson, AZ, USA

Patrick R. Durrell

Department of Astronomy and Astrophysics, Pennsylvania State University, University Park, PA, USA

J. Christopher Mihos

Department of Astronomy, Case Western Reserve University, Cleveland, OH, USA, USA

\begin{abstract}
We present the results from multiple surveys for intracluster planetary nebulae (IPNe) in nearby galaxy clusters and groups. We find that in the case of clusters, our observations imply: 1) the amount of intracluster starlight is significant, up to $20 \%$ of the total starlight, 2) in Virgo, is elongated along our line of sight, and 3) is clustered on the sky, implying ongoing tidal stripping. In contrast, searches for IPNe in groups have found little or no intra-group population, implying there may be something in the cluster environment that significantly enhances intracluster star production. From high-resolution N-body simulations, we find that the IPNe should create observable features in position-velocity space, and that these features may eventually allow us to place limits on the dynamics of galaxy clusters.
\end{abstract}

\section{Introduction}

The study of intracluster starlight (ICL) has grown dramatically in the last few years. Once thought to be just another oddity of Zwicky (1951), intracluster starlight may be a useful tool in understanding the evolution of galaxies in clusters, and may be an important chain in the recycling of intergalactic and interstellar matter (see Arnaboldi, this conference for a review). 
In particular, intracluster planetary nebulae (IPNe) are an excellent tracer of the intracluster light, and can be detected relatively easily in nearby galaxy clusters with deep narrow-band imaging. Extragalactic planetaries appear as point sources through a [O III] $\lambda 5007$ narrow-band filter, but disappear altogether when imaged through a moderate-band "off-band" exposure. Planetary nebulae also follow the [O III] $\lambda 5007$ planetary nebulae luminosity function (PNLF), which is a highly accurate distance indicator (see Ciardullo et al. 2002b and references therein). The well-defined luminosity function allows us to gather depth information on the intracluster stars. Finally, since IPNe are emissionline objects, their radial velocities can be determined with moderate-resolution spectroscopy (Freeman et al. 2000), and crucial dynamical information of the ICL gained.

Here, we focus on our group's efforts to search for IPNe in nearby galaxy clusters and groups, and give a brief summary on the results to date. The study of IPNe is still in its infancy, and much more can be learned. It is important to stress that surveys for IPNe are not pristine: we estimate that about $20 \%$ of our IPNe candidates in Virgo are actually Lyman- $\alpha$ galaxies at $\mathrm{z}=3.1$ (Ciardullo et al. 2002a).

\section{Studies in Clusters}

IPNe were first discovered in the Virgo cluster by Arnaboldi et al. (1996), and subsequently in the Fornax cluster by Theuns \& Warren (1997). Additional evidence for large numbers of IPNe in front of the Virgo elliptical M 87 quickly followed (Ciardullo et al. 1998). With the advent of wide-field mosaic detectors, it became feasible to observe much larger portions of the Virgo and Fornax clusters for IPNe.

Figure 1 shows the status of our surveys to date. We have detected 318 IPNe candidates in the Virgo cluster (Feldmeier, Ciardullo, \& Jacoby 1998; Feldmeier et al. 2003; Feldmeier et al. 2004), and 95 candidates in the Fornax cluster (Ciardullo et al. 2004). Transforming the numbers of IPNe to a stellar luminosity is complicated by several factors: the amount of background contamination, the known density differences of PNe to stellar luminosity (Ciardullo 1995), and any line-of-sight effects (Feldmeier et al. 2004). However, if we take conservative limits for such effects, we find that both clusters contain 10-20\% of their stars in an intracluster component.

The spatial distribution of IPNe in galaxy is also of great interest, since PNe closely follow the starlight in galaxies (Ciardullo et al. 1989). First, using the PNLF, we find the upper limit distance to each of our IPNe fields, and those of others (Arnaboldi et al. 2002; Okamura et al. 2003). We compare these distances to PNLF distances to ellipticals (Jacoby, Ciardullo, \& Ford 1990; Ciardullo et al. 1998), and HST observations of Cepheids in Virgo spirals (Freedman et al. 2001). We find that the IPNe are enormously extended, up to $3 \mathrm{Mpc}$ in depth (see Figure 2). This in agreement with the depth seen in Virgo through Tully-Fisher observations of Virgo spirals, indicating that the bulk of the IPNe comes from late-type galaxies.

When we compare the IPNe density to that of the galaxies directly, we find that in both clusters, the amount of IPNe may drop more slowly with radius 

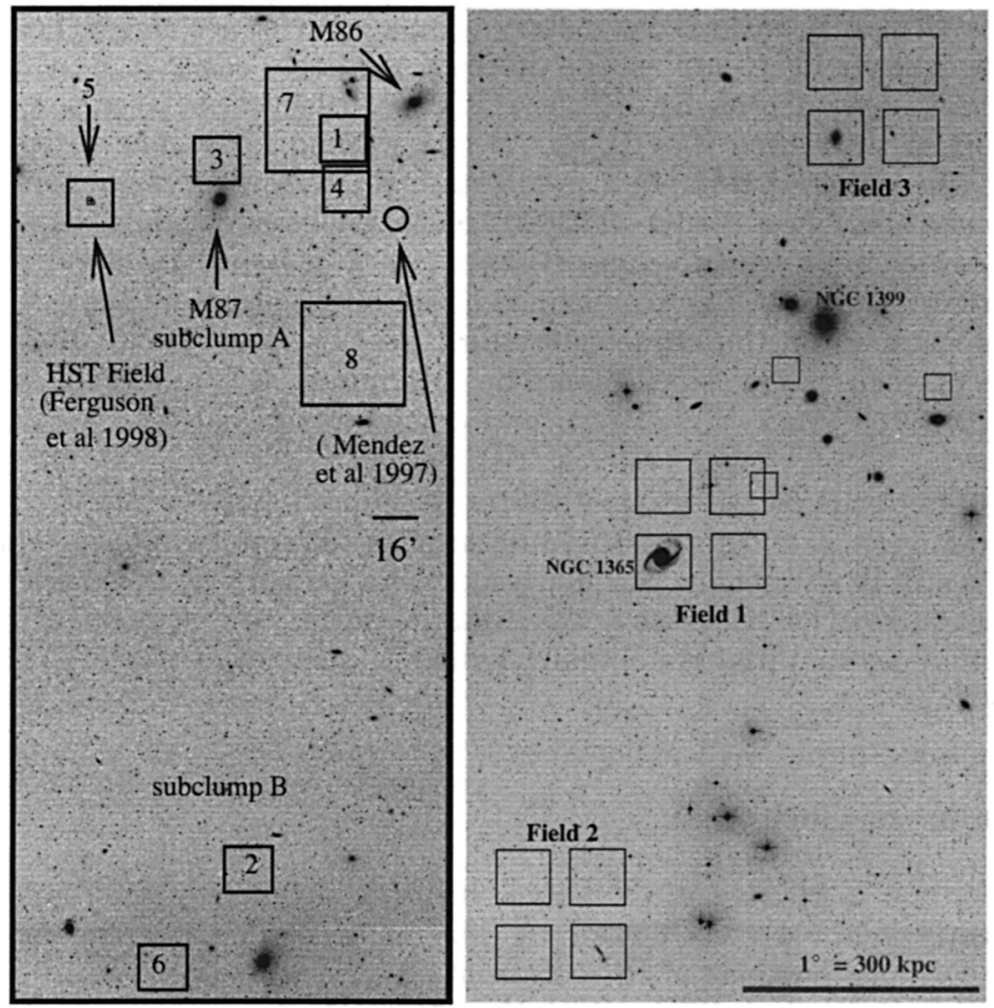

Figure 1. Regions of the Virgo (left) and Fornax (right) clusters, drawn from the Digital Sky Survey, with the location of our IPN survey fields marked. Arrows represent additional detections of ICL made by other researchers. Thus far, we have found a total of 413 IPN candidates in both clusters, but we have observed less than $1 \%$ of each cluster.

than that of the galaxies, though the scatter is still large due to small numbers. From observing the positions of the IPNe on the sky, we also gain some insight on the spatial distribution of the ICL. We find that the IPNe are clustered on arcminute scales, implying that there is ongoing tidal stripping in these clusters.

\section{Studies in Groups}

Although the amount of intracluster starlight in clusters such as Virgo and Fornax is now well established, the amount of 'intra-group' starlight is much less clear. Theoretical studies predict that if most intracluster stars are removed by galaxy collisions (e.g. Richstone \& Malumuth 1983; Moore et al. 1996), the fraction of intra-group stars, to first order, should be a smooth function of galaxy number density $\left(\mathrm{L}_{I C L} \sim \mathrm{N}_{\text {Gal }}^{2}\right)$. 


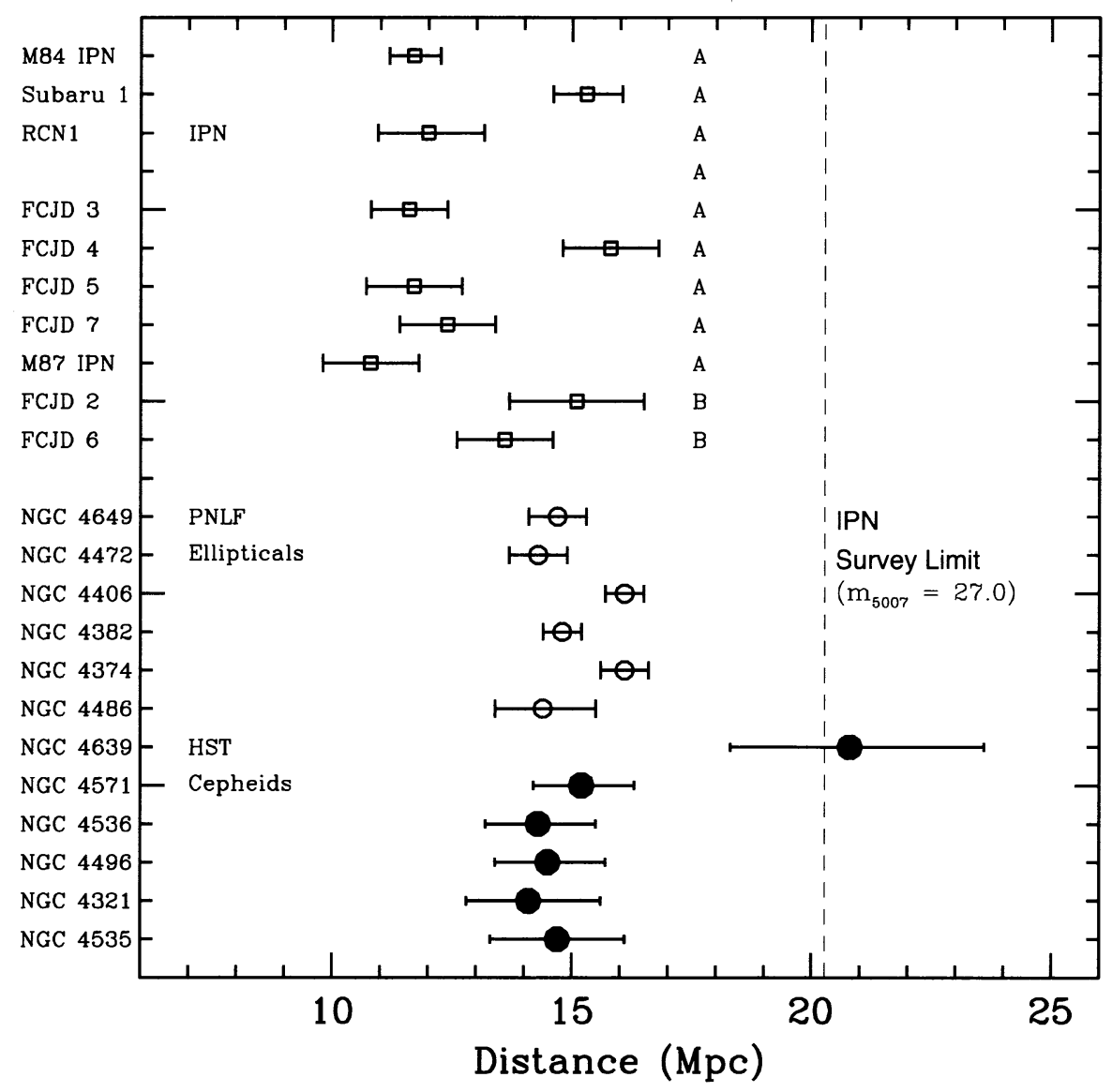

Figure 2. The upper-limit distances to our and other IPNe fields compared to PNLF distances to elliptical galaxies, and HST observations of Cepheids in Virgo spiral galaxies. As can be clearly seen, some of the IPNe fields are up to $3 \mathrm{Mpc}$ in front of the core of the Virgo cluster 
To test this hypothesis, we have undertaken a large-scale [O III] $\lambda 5007$ IPN survey of the nearby M 81 group of galaxies. This galaxy group is known to be strongly interacting, with multiple tidal tails seen in $\mathrm{H}$ I gas (Yun, Ho, \& Lo 1994). We have surveyed 1.44 square degrees of this system with the KPNO 4-m and the Mosaic camera, and have reached at least two magnitudes down the PNLF in all of our fields.

Although the analysis is ongoing, there is already a clear result: there is substantially less IPNe in the M 81 group than in the rich clusters. For example, if we take the results from our Field 1, we find 102 PNe candidates near M81 proper, but no objects whatsoever in the remaining half of the field. If we assume this density limit is typical, this leads us to a intra-group fraction of $1.3 \%$. These results are strengthened by a deep broad-band survey of the M81 group to look for intra-group red giant stars, which find similar small limits (see Durrell, this volume).

Our result, combined with the similar result (1.6\%) for the Leo I group by Castro-Rodríguez et al. (2003), strongly implies there is substantially less (4-15 times less) intra-group starlight in groups as there is in galaxy clusters. The drop in density is unexpected, and will need to be explained by models of intracluster star production (see Ciardullo, this volume).

In both cases, at least one of the motivations for searching both of these particular galaxy groups for intra-group PNe was the disturbed H I morphologies previously known in Leo I and M81 (Schneider et al. 1989; Yun, Ho, \& Lo 1994). This property seems be somewhat misleading in the case of an older stellar IPNe population. This may simply be that in normal spiral galaxies, the H I distribution would be removed first in galaxy interaction, before any stars could escape due to its larger mean radius. Observations of at least one undisturbed galaxy group for IPNe would be helpful to ensure this result.

\section{Dynamical Studies of Intracluster Light}

Since there are already large samples of IPNe candidates available that can have their radial velocities determined, and larger IPNe samples will be forthcoming, it is important to understand the velocity strucure of the ICL. Several groups of researchers have begun the process of modelling the velocity structure of the ICL through a variety of simulations (Moore et al. 1996; Dubinski, Murali, \& Ouyed 2001; Napolitano et al. 2003). In these models, the intracluster light generally follows a radial orbit distribution, but is dynamically unrelaxed, and fills the observed phase space non-uniformly, due to the presence of tidal debris. The radial orbit envelope may allow us to independently estimate the mass profile of galaxy clusters (Dubinski, Murali, \& Ouyed 2001), independent of more traditional methods. Moreover, since the phase-space clumpiness of the ICL is related to the cluster's dynamical age, the simulations, combined with the IPN observations, will allow us to place interesting limits on this quantity for the clusters observed, as well as potential limits on cosmological parameters.

To confirm these results, we have constructed our own high-resolution, fully self-consistent $\mathrm{N}$-body models, using large-scale structure simulations to find clusters at high redshift. We then replace these low resolution galaxies with selfconsistent models of galaxies (Hernquist 1993), and run the simulations. We 
confirm the general results thus far: our ongoing focus is on developing useful metrics for observations.

\section{References}

Arnaboldi, M. et al. 1996, ApJ, 472, 145

Arnaboldi, M. et al. 2002, AJ, 123, 760

Castro-Rodríguez, N., et al. 2003, A\&A, 405, 803

Ciardullo, R. 1995, in IAU Highlights of Astronomy, 10, ed. I. Appenzeller (Dordrecht: Kluwer), p. 507

Ciardullo, R., Jacoby, G.H. \& Ford, H.C. 1989, ApJ344, 715

Ciardullo, R., et al. 1998, ApJ, 492, 62

Ciardullo, R., et al. 2002a, ApJ, 566, 784

Ciardullo, R., et al. 2002b, ApJ, 577, 31

Dubinski, J., Murali, C., \& Ouyed, R. 2001, unpublished preprint

Feldmeier, J. J., Ciardullo, R., \& Jacoby, G. H. 1998, ApJ, 503, 109

Feldmeier, J. J., et al. 2003 ApJS, 145, 65

Feldmeier, J. J., et al. 2004 ApJ, in prep

Freedman, W. L. et al. 2001, ApJ, 553, 47

Freeman, K. C. et al. 2000, ASP Conf. Ser. 197, (San Francisco: ASP) 389.

Hernquist, L. 1993, ApJS86, 389

Jacoby, G. H., Ciardullo, R., \& Ford, H. C. 1990, ApJ, 356, 332

Moore, B., et al. 1996, Nature, 379, 613

Napolitano, N.R., et al. 2003, ApJ, 594, 172

Okamura, S., et al. 2003, PASJ, 54, 883

Richstone D. O., \& Malumuth, E.M. 1983, ApJ, 268, 30

Schneider, S. et al. 1989, AJ97, 666

Theuns, T., \& Warren, S. J. 1997, MNRAS, 284, L11

Yun, M. S., Ho, P. T. P., \& Lo, K. Y. 1994, Nature, 372, 530

Zwicky, F. 1951, PASP, 63, 61 